\title{
Polarization dynamics of a multimode vertical-cavity surface-emitting laser subject to orthogonal optical injection
}

\author{
Hong Lin, ${ }^{1, *}$ Yu Zhang, ${ }^{1}$ David W. Pierce, ${ }^{1}$ Ana Quirce, ${ }^{2,3}$ and Angel Valle ${ }^{2}$ \\ ${ }^{1}$ Department of Physics and Astronomy, Bates College, Lewiston, Maine 04240, USA \\ ${ }^{2}$ Instituto de Fisica de Cantabria (CSIC-Univ. Cantabria), Avda, Los Castros s/n, E39005 Santander, Spain \\ ${ }^{3}$ Dept. de Fisica Moderna, Fac. Ciencias, Univ. Cantabria, Avda, Los Castros s/n, E39005 Santander, Spain \\ *Corresponding author: hlin@bates.edu
}

Received November 4, 2011; revised December 27, 2011; accepted January 3, 2012; posted January 3, 2012 (Doc. ID 157680); published March 30, 2012

\begin{abstract}
We have studied the effect of orthogonal optical injection on a multitransverse-mode vertical-cavity surfaceemitting laser (VCSEL). The injection beam comes from a similar VCSEL. Our results reveal that, when the receiver operates with several strong transverse modes of parallel polarization, optical injection can induce polarization switching in all of them while only one mode is locked to the external frequency. The induced switching can occur for a very weak (a few microwatts) injected beam. Periodic oscillations and low-frequency irregular pulsations are observed as well. @ 2012 Optical Society of America
\end{abstract}

\section{INTRODUCTION}

Optical injection on vertical-cavity surface-emitting lasers (VCSELs) has been an active research area since 1990's [1-19]. Optical injection can induce frequency locking and rich nonlinear phenomena including enhanced relaxation oscillation [1] ], bistability [2] ], nondegenerate four-wave mixing [5] , subharmonic generation [미, $\underline{3}, \underline{10}]$, and chaotic dynamics $[\underline{6}, \underline{10}]$. With optical injection, it is possible to reduce chirp and nonlinearity [7] and to achieve all-optical inverting via frequency locking [8]. Depending on the polarization of the injected signal relative to the polarization of receiver, two configurations have been used in the study: parallel injection and orthogonal injection. Parallel injection means that polarization of the injected signal is parallel to the polarization of the receiver; orthogonal injection refers to the case in which the polarization of the injection is perpendicular to that of the receiver. However, polarization of a VCSEL is not always stable. It may vary with the operating temperature and bias current of the VCSEL [20]. Besides, higher-order transverse modes can start oscillation when the bias current is increased, and adjacent transverse modes have parallel or orthogonal polarizations [21]. The complexity in polarization and transverse mode operation increases richness of dynamics when optical injection is applied to a VCSEL. In recent years, single-frequency optical injection has been studied in twotransverse-mode VCSELs both theoretically and experimentally $[\underline{4}, \underline{5}, \underline{8}, \underline{9}, \underline{11}, \underline{15}, \underline{18}]$. It has been observed that optical injection can induce polarization switching (PS) [11], mode switching [8,11], and bistability [18] via frequency locking between one frequency of the VCSEL and the injected beam. Nondegenerate four-wave mixing [5] , antiphase dynamics and chaotic oscillation $[\underline{4}, \underline{15}]$ are obtained outside the locking regime. Very recently, we studied experimentally two-frequency orthogonal optical injection on a multimode
VCSEL. We obtained complete polarization switching when the two dominant modes are locked to the corresponding modes of the injected light [19]. The interests in effects of optical injection on VCSELs arise from both potential applications and intellectual curiosity for mechanisms underlying the phenomena. For example, control of polarization is an important issue in telecommunication applications because coupling of a VCSEL to an optical fiber may result in different loss for the two polarizing modes and lead to an increase of the intensity noise at the fiber output [10]. On the other hand, a well-controlled polarization switching and polarization bistability may be useful for all-optical signal processing functionalities $[\underline{8}, \underline{18}]$. From the point of view of fundamental sciences, the interplay of polarization and transverse modes in optically injected VCSELs still needs investigating.

In this paper we are reporting our experimental investigation on the polarization dynamics of a multitransverse-mode VCSEL subject to a single-frequency orthogonal optical injection. Our work differs from the previous ones in the following aspects. First, our VCSEL operates with more than two transverse modes and demonstrates unique behaviors under optical injection. In the previous studies [5], it was shown that transverse modes of parallel polarization behave independently of each other for both parallel and orthogonal optical injection. On contrary, we have observed simultaneous polarization switching of several $X$-polarized transverse modes when one of the modes is frequency-locked to the injected beam even when the injection power is only a few microwatts. This opens up the possibility of controlling the polarization of the VCSEL in the multitransverse-mode regime. Second, while most experiments use a tunable semiconductor laser as the transmitter, our transmitter is a VCSEL that is very similar to the receiver: they are from the same manufacturer, of the same model number, and came in same package. Given the recent efforts in studying VCSEL-by-VCSEL optically 
injection-locked systems [1]], it is necessary to understand polarization dynamics in such a system.

\section{EXPERIMENTAL SETUP AND CHARACTERISTICS OF THE RECEIVER}

In our experiment, a proton-implanted VCSEL, $V_{1}$ (receiver), emitting at $847 \mathrm{~nm}$ receives a single-frequency injected signal provided by another VCSEL, $V_{2}$ (transmitter), of the same model, as shown in Fig. 1. The temperatures of the two VCSELs are stabilized at $24.01{ }^{\circ} \mathrm{C}$ and $32.02{ }^{\circ} \mathrm{C}$ separately by temperature controllers of same model (Thorlabs TEC2000). Their bias currents are controlled by current drivers (Thorlabs LDC200C) with accuracy $0.001 \mathrm{~mA}$. The dominant polarization of both VCSELs is parallel to the optical table, which is termed $X$ polarization. The polarization perpendicular to the optical table is termed $Y$ polarization. The $X$ polarization of the transmitter is selected by a polarizing beam splitter (PBS) and sent through an optical isolator (ISO). Since the isolator makes the polarization rotated by $45^{\circ}$, a half-wave plate (HWP) is placed behind the isolator to rotate the polarization of the transmitted light by another $45^{\circ}$, becoming perpendicular to the optical table. Now the polarization of the injected light is orthogonal to the dominant polarization of the receiver. The injection is sent into the receiver by mirror $M_{2}$ and a nonpolarizing plate beamsplitter $M_{1}$. We obtain the optimal alignment when the injection power necessary to induce polarization switching is minimized at the boundary of the PS region.

For the purpose of observation and measurement, the output of $V_{1}$ is split at $M_{1}$ : the transmitted part is sent to a Fabry-Perot (F-P) spectrum analyzer (FSR $150 \mathrm{GHz}$ ) and a charge coupled device (CCD) camera, the reflected light is sent to a one-meter spectrometer (Jobin Yvon $1000 \mathrm{~m}$ ) and a fast detector (Newport 1580B, $12 \mathrm{GHz}$ ) which can be connected to an RF spectrum analyzer (Agilent EXA N9010A, $9 \mathrm{kHz}$ to $26.5 \mathrm{GHz}$ ) or a digital oscilloscope (Tektronix DPO 7254, $2.5 \mathrm{GHz}$ ). With the aid of another PBS and a half-wave plate, we can detect spectrum and dynamics of each polarization of $V_{1}$ as well as its power. The power of each polarization is measured with a power meter. We can also set up

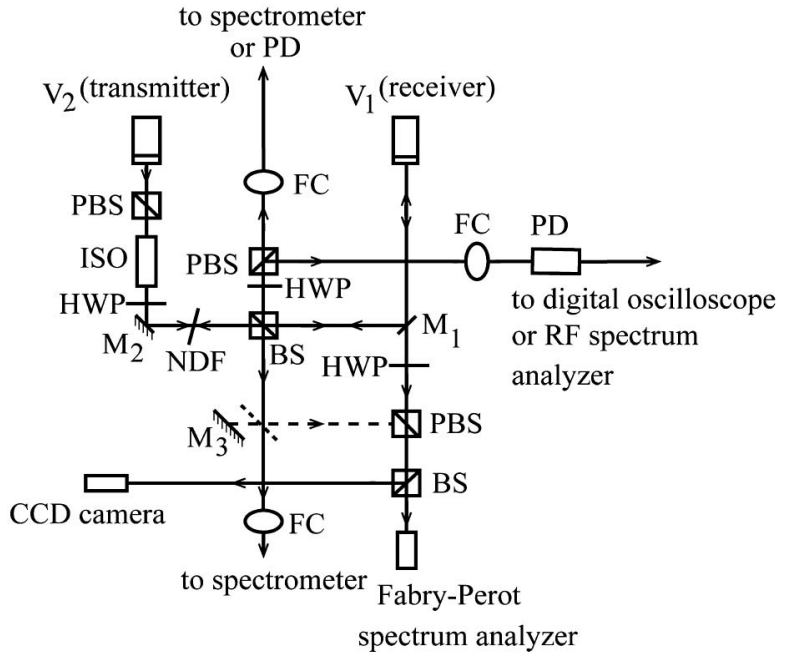

Fig. 1. Experimental setup, where PBS stands for polarizing beamsplitter, BS for nonpolarizing beamsplitter, HWP for half-wave plate, ISO for optical isolator, FC for fiber coupler, and PD for fast photodetector. $M_{1}$ is a plate beamsplitter, $M_{2}$ and $M_{3}$ are mirrors. two fast detectors to observe temporal behaviors of the $X$ and $Y$ polarizations simultaneously. Half of the beam from the transmitter $V_{2}$ can be sent to the spectrometer or to the F-P spectrum analyzer and the CCD camera. In order to send the light of $V_{2}$ to the $\mathrm{F}-\mathrm{P}$ spectrum analyzer, we use a mirror, $M_{3}$, installed on a translational stage. $M_{3}$ and the PBS that reflects the output of $V_{2}$ are carefully aligned to get the frequency structure of $V_{2}$ at the F-P spectrum analyzer. The frequency (wavelength) detuning is obtained from the F-P spectrum analyzer (spectrometer). The neutral density filter (NDF) is used to adjust the injection power. The power of the injected signal is measured in front of the collimating lens of $V_{1}$.

As shown in Fig. 2(a), the solitary $V_{1}$ is dominantly polarized in the $X$ direction and no polarization switch occurs. From threshold to $3.33 \mathrm{~mA}$, the VCSEL operates in fundamental mode $\left(\mathrm{TEM}_{00}\right.$ mode). The second mode, a higher-order transverse mode, starts lasing from $3.33 \mathrm{~mA}$. Its beam profile indicates that it can be described as a $\mathrm{TEM}_{01}$ mode. From $3.65 \mathrm{~mA}$ to $4.75 \mathrm{~mA}$, the VCSEL operates with three transverse modes. The third mode is a $\mathrm{TEM}_{10}$ mode. The fourth transverse mode can be described as a $\mathrm{TEM}_{02}$ mode. It is on from $4.75 \mathrm{~mA}$ and is essentially $Y$ polarized. In our study, we focused on the bias current between $4.8 \mathrm{~mA}$ and $5.6 \mathrm{~mA}$, where the receiver operates with four transverse modes.

The polarization feature of $V_{1}$ and the spatial profile of each transverse mode are shown in Fig. 2(b). The fundamental mode and the $\mathrm{TEM}_{10}$ mode are $X$ polarized; however, each has a nonlasing $Y$ mode with a higher frequency. The fourth mode $\left(\mathrm{TEM}_{02}\right)$ is $Y$ polarized. The $\mathrm{TEM}_{01}$ mode has two polarized states: an elliptically polarized state and a nonlasing $Y$ polarized state. The frequency of the $Y$ polarization is $8 \mathrm{GHz}$ higher. As illustrated, the $X$ component of the elliptical polarization is much stronger than its $Y$ component, therefore
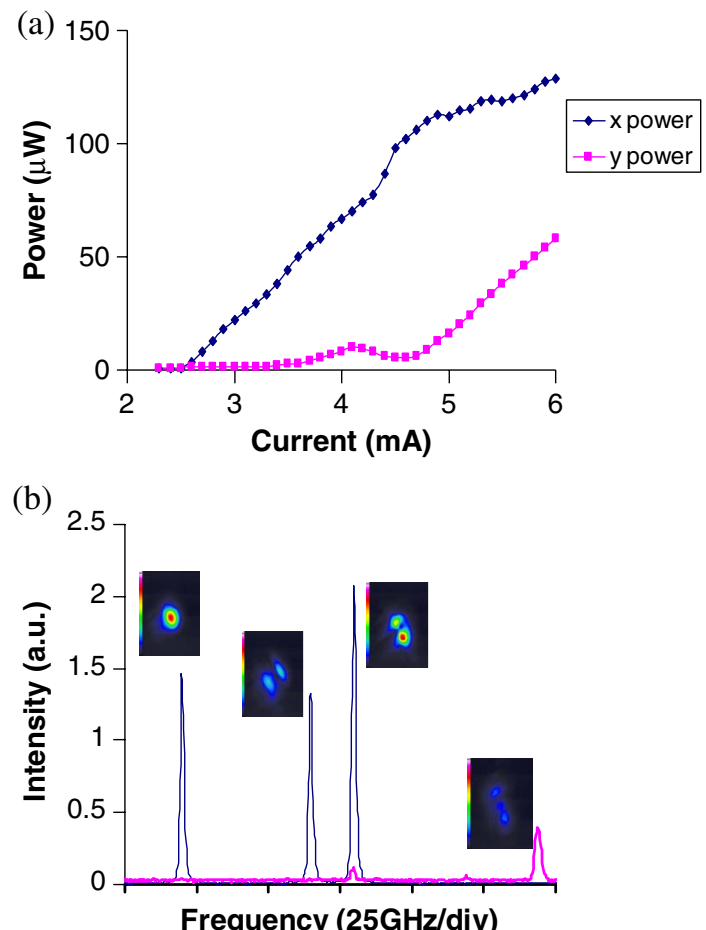

Fig. 2. (Color online) Polarization-resolved (a) L-I curves and (b) optical spectra of the solitary $V_{1}$. The bias current of $V_{1}$ is $5.219 \mathrm{~mA}$. (Blue: $X$ polarization; pink: $Y$ polarization.) 
this polarization can be regarded as $X$ polarization essentially. The frequency difference is $45 \mathrm{GHz}$ between the $\mathrm{TEM}_{00}$ and $\mathrm{TEM}_{10}$ mode and $60 \mathrm{GHz}$ between the $\mathrm{TEM}_{00}$ and essentially $X$-polarized $\mathrm{TEM}_{01}$ mode. The frequency of the $Y$-polarized $\mathrm{TEM}_{02}$ mode is $124 \mathrm{GHz}$ higher than the $\mathrm{TEM}_{00}$ mode.

\section{EXPERIMENTAL RESULTS}

In our experiment, the bias current of $V_{2}, I_{2}$, is $3.100 \mathrm{~mA}$. The output from $V_{2}$ is single frequency with the beam profile of the fundamental mode. We tune $I_{1}$, the bias current of the receiver, to change the frequency detuning, $\Delta \nu$, between $V_{1}$ and $V_{2}$. We define $\Delta \nu$ as the frequency difference between the injected light, $\nu_{2}$, and the nonlasing $Y$-polarized TEM $_{01}$ mode of the solitary $V_{1}, \nu_{1 Y}$. The frequency detuning is read from the optical spectra obtained from the F-P spectrum analyzer. The reading uncertainty is $\sim 0.6 \mathrm{GHz}$. Another control parameter is the injection power, $P_{\text {inj. }}$. The injection power is measured in front of the collimating lens of $V_{1}$. Its value is adjusted by using a neutral density filter (NDF). The maximum injection power is $19.6 \mu \mathrm{W}$.

Figure 3 demonstrates polarization switching in the total output power when the injection power is varied and frequency detuning is relatively small. In Fig. 3(a), dominantly $X$-polarized total power switches to $Y$-polarized power when $P_{\text {inj }}$ is greater than $10 \mu \mathrm{W}$. The corresponding frequency detuning is $\Delta \nu=-2.0 \mathrm{GHz}$. When $\Delta \nu=-1.4 \mathrm{GHz}$, polarization switching occurs for even lower injection power. This is similar to $[\underline{10}, 11,17]$, in which a minimum injection power induces PS for appropriate detuning. However, the power of $Y(X)$ polarization is lower (higher) than that in Fig. 3 (a) by approximately $15 \mu \mathrm{W}$. This is because the transverse modes do not always have PS together. As shown in Fig. 4,
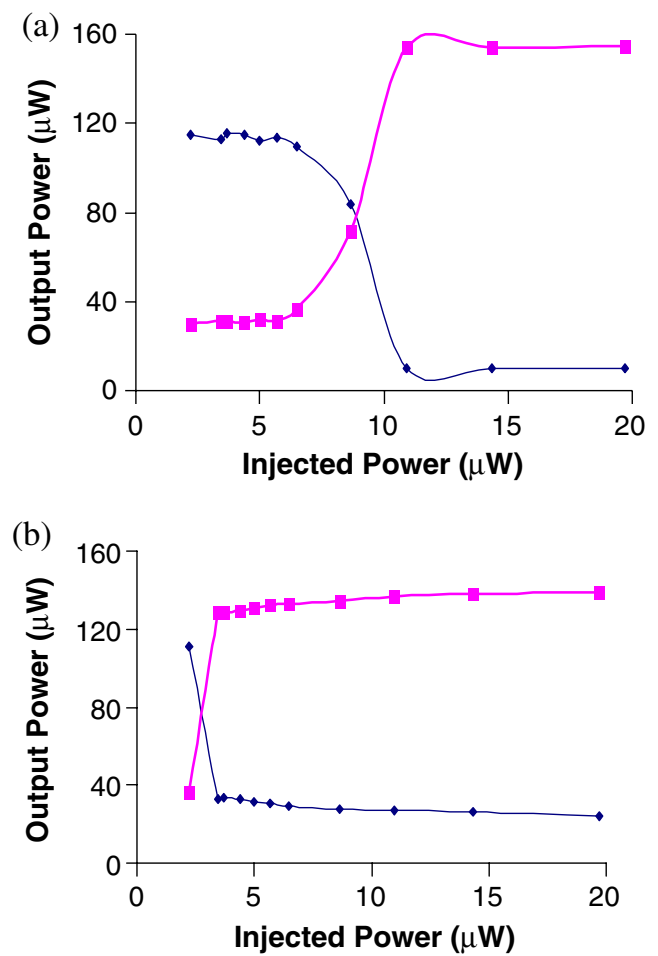

Fig. 3. (Color online) Polarization-resolved total power versus $P_{\text {inj }}$ for (a) $\Delta \nu=-2.0 \mathrm{GHz}$ and (b) $\Delta \nu=-1.4 \mathrm{GHz}$. (Blue diamond: $X$ polarization; pink square: $Y$ polarization.)
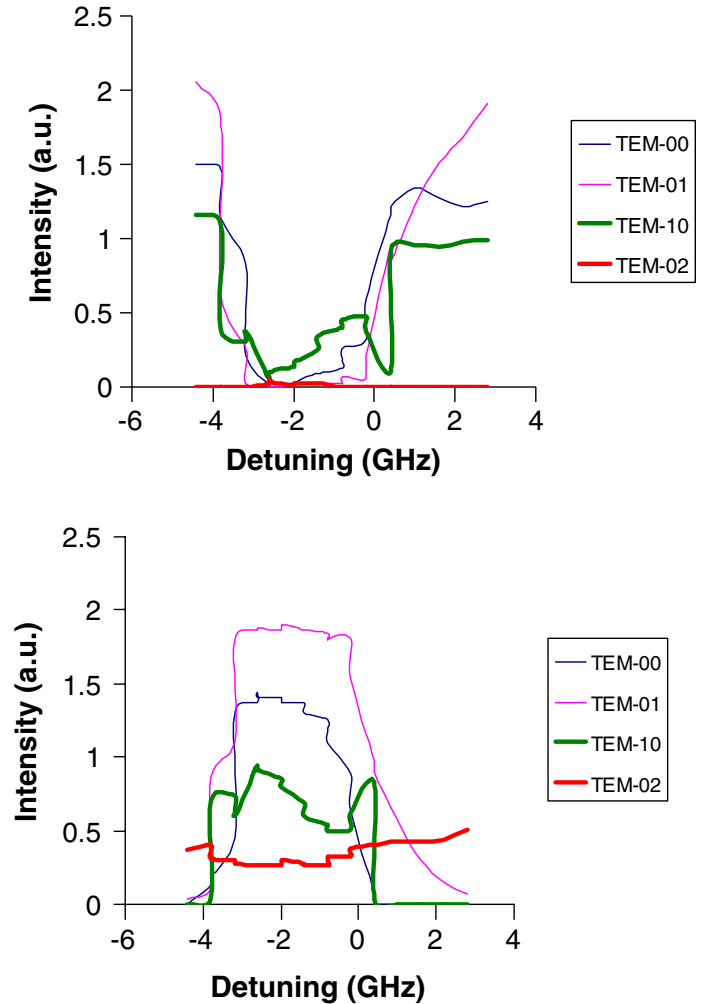

Fig. 4. (Color online) (a) $X$-polarized and (b) $Y$-polarized modal intensities versus frequency detuning, where the injection power is $19.6 \mu \mathrm{W}$.

though the three $X$-polarized modes start to drop simultaneously, they do not reach their lowest intensity together. Among the three modes, the $\mathrm{TEM}_{01}$ mode undergoes polarization switching in a detuning range of $\sim 3 \mathrm{GHz}$. The other two, the $X$-polarized fundamental mode and $\mathrm{TEM}_{10}$ mode, stay at their lowest intensity for a very narrow range of detuning and then begin to increase. Therefore, though the total power still undergoes polarization switching in Fig. 3(b), the switching is not as complete as in Fig. 3(a). The modal intensity also shows that the influence of the injection on the fourth transverse mode is minimal: its $Y$-polarized state only has a slight decrease and the $X$ polarization increases slightly when the detuning is changed from $-3 \mathrm{GHz}$ to $0 \mathrm{GHz}$.

The polarized optical spectra of $V_{1}$ in the PS region are given in Fig. 5. It is obvious that frequency locking is achieved between the $\bar{Y}$-polarized $\mathrm{TEM}_{01}$ mode and the injected beam. Frequencies of the $Y$-polarized fundamental mode and $\mathrm{TEM}_{10}$ mode are $\sim 7 \mathrm{GHz}$ higher than their $X$-polarized counterparts, indicating that their nonlasing $Y$-polarizations start oscillation now. Their frequencies, however, are far from that of the injection. Thus it is reasonable to say that the underlying physical mechanism is not frequency locking for the fundamental mode and $\mathrm{TEM}_{10}$ mode. Note that the $Y(X)$ polarization of the $\mathrm{TEM}_{02}$ mode has a slight decrease (increase). This agrees with what is shown in Fig. 4 .

As a comparison, we give the polarized optical spectra of $V_{1}$ when the injected signal has frequency locking with the fundamental mode and the $\mathrm{TEM}_{10}$ mode, respectively. Figures $\underline{6(\mathrm{a})}$ and $6(\mathrm{~b})$ show injection-induced polarization switching in the $\mathrm{TEM}_{00}$ mode for $I_{1}$ to be $3.408 \mathrm{~mA}$. For this bias current, the $\mathrm{TEM}_{01}$ mode has started oscillation but is 

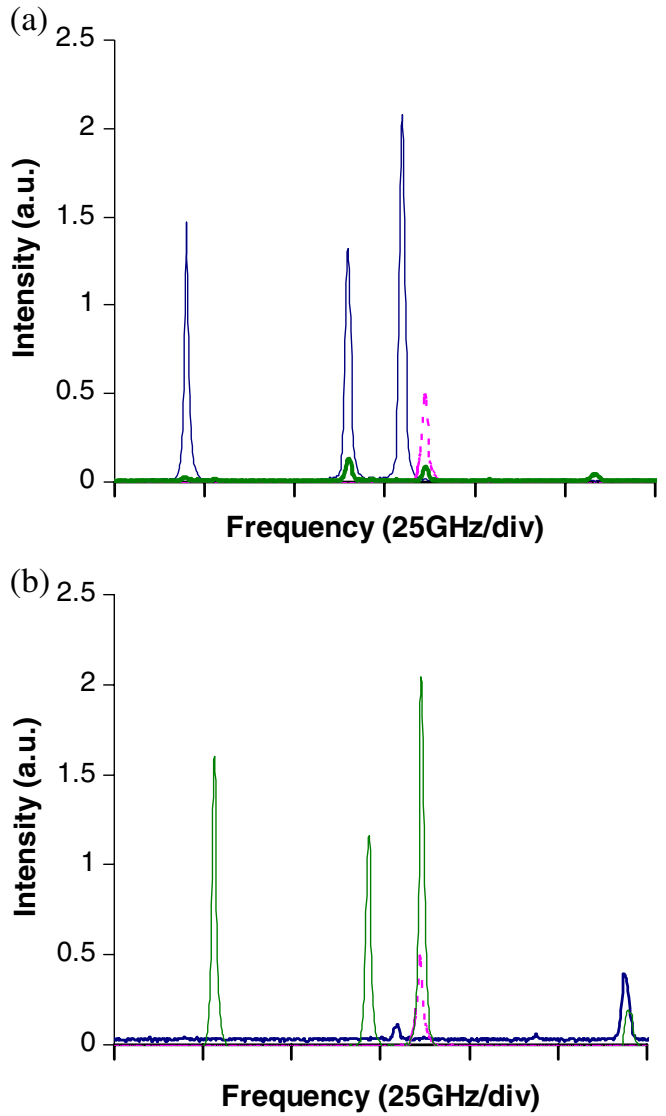

Fig. 5. (Color online) Polarization-resolved optical spectra of $V_{1}$ for $\Delta \nu$ to be $-2 \mathrm{GHz}$ and $P_{\text {inj }}$ to be $19.5 \mu \mathrm{W}$. (a) $X$ polarization (blue: solitary $V_{1}$; green: $V_{1}$ with injection) and (b) $Y$ polarization (blue: solitary $V_{1}$; green: $V_{1}$ with injection). The pink, dashed curve represents injected signal in both polarized spectra.

much weaker than the fundamental mode. The frequency detuning in Figs. 6(a) and 6(b) is $-67 \mathrm{GHz}$. It is obvious that the fundamental mode becomes $Y$-polarized and frequency locking is achieved between the injected signal and the $Y$-polarized fundamental mode. The $\mathrm{TEM}_{01}$ (the weak peak in Fig. 6(a)) is not affected by the injection. This is somewhat similar to the behavior of the $\mathrm{TEM}_{02}$ mode in Fig. 5, in which the three strong $X$-polarized modes undergo polarization switching whereas the $\mathrm{TEM}_{02}$ mode only has a slight change in its intensity. When the injection gets close to the $\mathrm{TEM}_{10}$ mode, it causes polarization switching in the $X$-polarized $\mathrm{TEM}_{10}$ mode but does not affect polarization of the other modes, as shown in Figs. 6(c) and $\underline{6(d)}$ for detuning to be $-16 \mathrm{GHz}$.

When the frequency detuning and injection power are varied separately, the output of the receiver demonstrates polarization switching and various instabilities. The dynamics of $V_{1}$ is mapped in Fig. 7. Experimentally, we consider that the mode switches from $X$ to $Y$ polarization when its $X$-polarized modal intensity drops to one-tenth of its original. Similarly, the mode is considered switching back when its $X$-polarized modal intensity rises to one-tenth of its original. The modal polarization switching, however, is complex. As shown in Fig. 4, the $\mathrm{TEM}_{10}$ mode switches twice as detuning is varied from $\sim-3 \mathrm{GHz}$ to $1 \mathrm{GHz}$. Therefore we define a modal polarization switching regime instead of illustrating switching/switching back boundary for each mode. The modal polarization
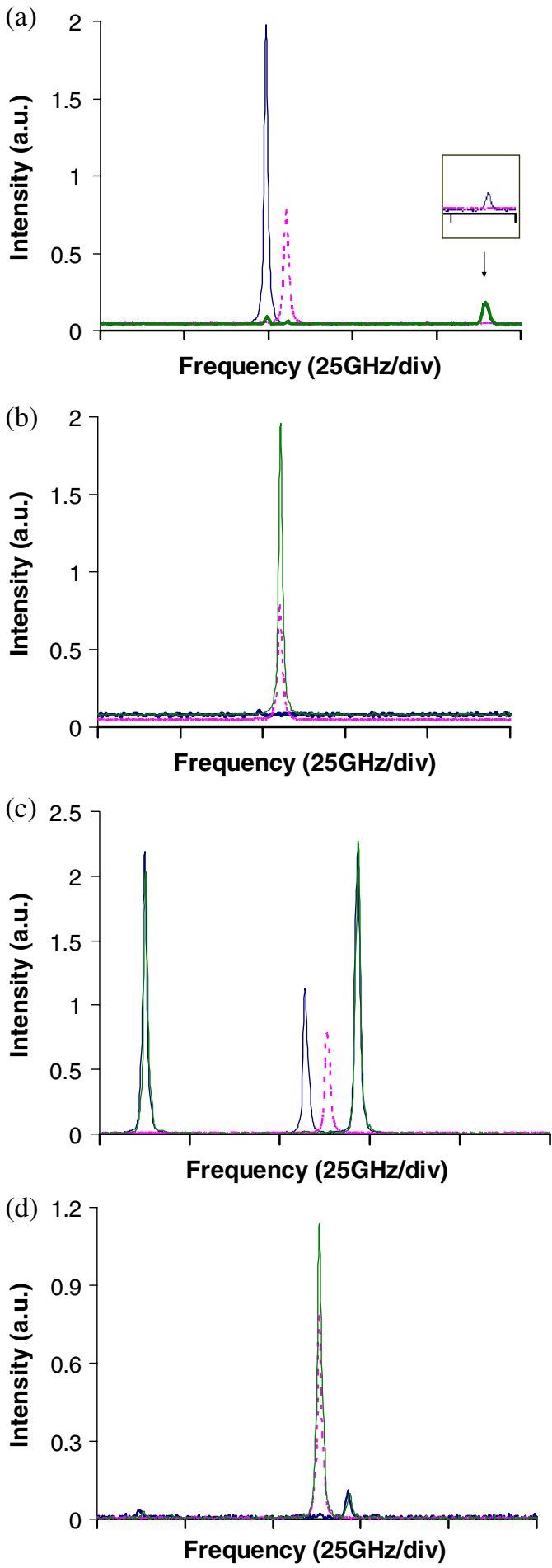

Fig. 6. (Color online) Polarization-resolved optical spectra of $V_{1}$ for (a,b) $I_{1}=3.408 \mathrm{~mA}, \Delta \nu=-67 \mathrm{GHz}$ and $(\mathrm{c}, \mathrm{d}) I_{1}=4.799 \mathrm{~mA}$, $\Delta \nu=-16 \mathrm{GHz} . P_{\text {inj }}$ is $19.5 \mu \mathrm{W}$. (a) and (c) give $X$ polarization (blue: solitary $V_{1}$; green : $V_{1}$ with injection; pink: injected signal). (b) and (d) are $Y$ polarization (blue: solitary $V_{1}$; green: $V_{1}$ with injection; pink dashed: injected signal). An inset is included in (a) to illustrate with clarity the $\mathrm{TEM}_{01}$ mode of the solitary $V_{1}$.

switching refers to the regime where there are one or more transverse modes switching from $X$-polarized state to $Y$-polarized state. As for the slight discrepancy between Fig. 4 and Fig. 7 (in Fig. 4, modal switching regime is from $\sim-3 \mathrm{GHz}$ to $\sim 0.5 \overline{\mathrm{GHz}}$ for maximum injection power; in Fig. 7 , the 


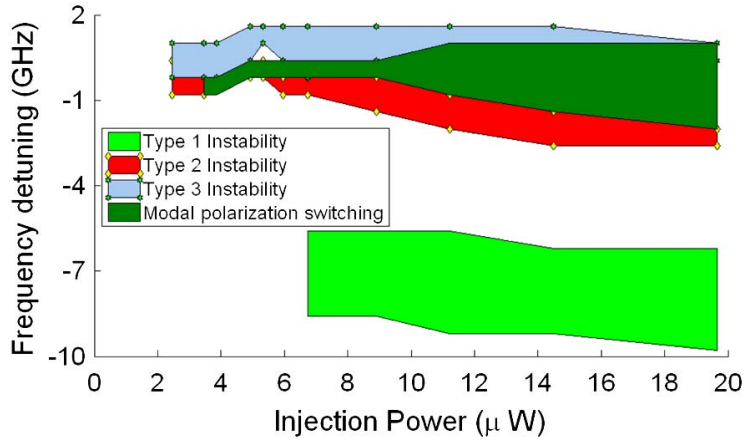

Fig. 7. (Color online) Dynamics map of $V_{1}$ in the parameter plane of detuning versus injection power, in which regions of modal polarization switching and three types of instabilities are illustrated, respectively.

modal switching regime is from $\sim-2 \mathrm{GHz}$ to $\sim 1.5 \mathrm{GHz}$ for maximum injection power), it is probably caused by uncertainties in reading and alignment of the F-P spectrum analyzer. The overall dynamics, however, repeats the same pattern as the detuning is tuned from negative value to positive. In the modal PS regime, when all the $X$-polarized modes have PS, the output is stable. Otherwise, a low-frequency $(<0.2 \mathrm{GHz})$ shoulder is observed in the RF power spectrum of the receiver, as shown in Fig. 8(a). The corresponding time series manifest antiphase, irregular fluctuations [Fig. 8(b))]. This is because modal polarization switch and switching back do not always occur simultaneously for all transverse modes in the modal polarization regime. When some modes switch whereas others do not, polarization competition will happen and results in irregular antiphase fluctuations of relatively low frequencies.

We analyzed the RF power spectrum of $V_{1}$ and categorized the instabilities into three types. In the analysis, we take a distinguishable peak in the power spectrum into account when the magnitude of the peak is equal or greater than $3 \mathrm{dBm}$. Among the three types of instabilities, type 1 and type 2 are also observed when the VCSEL is subject to a twofrequency optical injection [19]. When the detuning is greater than $-10 \mathrm{GHz}$, a sharp peak is observed in the power spectrum and its frequency changes with the detuning. We term this type of oscillation as type 1 instability. As shown in Fig. 9 (a), there is a sharp peak of $\sim 0.8 \mathrm{GHz}$ in the RF power spectrum of $V_{1}$ for detuning to be $-7.4 \mathrm{GHz}$. For this specific detuning, the frequency difference between the injection and the elliptically polarized $\mathrm{TEM}_{01}$ mode is $0.6 \mathrm{GHz}$. These two frequencies are the same by taking the reading uncertainty into account. On the contrary, type 1 instability is not observed when the external signal is injected to the $\mathrm{TEM}_{00}$ or $\mathrm{TEM}_{10}$ mode of the receiver because these two modes are $X$-polarized in solitary operation. Therefore the oscillation is probably the beating of the injected beam and the $y$-component of the $\mathrm{TEM}_{01}$ mode. The second type of instability is represented by a peak $\sim 1$ to $2 \mathrm{GHz}$ in the power spectrum and a low-frequency enhancement of the power spectrum of the $Y$-polarization, as shown in Fig. 9(b). It occurs immediately before a mode switches from $X$ to $\overline{Y \text { polarization }}$ and the bandwidth of the frequency peak in the power spectrum is wider than that in type 1 instability. The origin of this instability is likely due to noise-induced mode hopping that typically accompanies polarization switching. Polarization mode hopping in quantum-well VCSELs is only observed when the laser is biased in the vicinity of the current of an abrupt PS
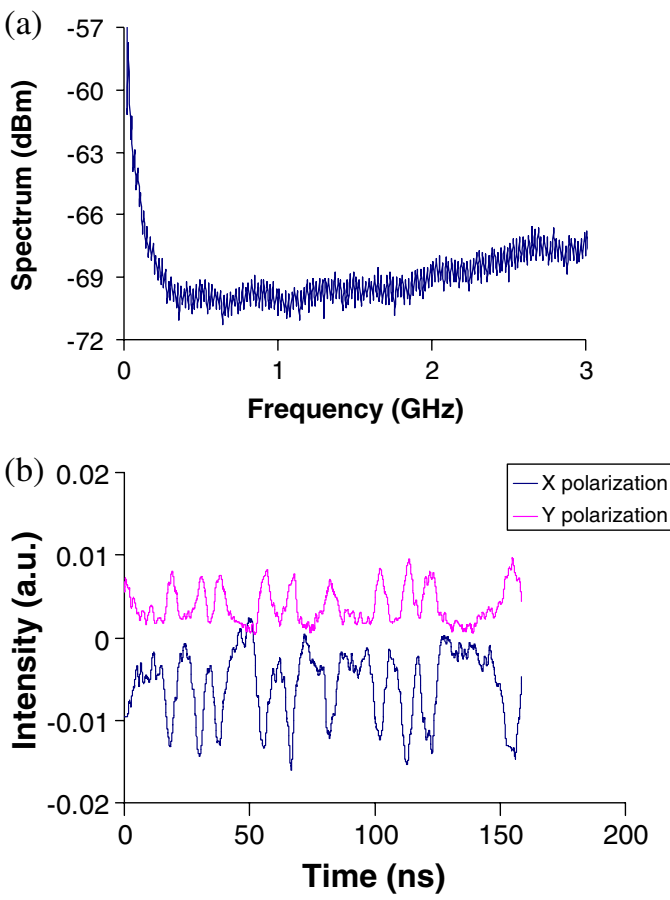

Fig. 8. (Color online) (a) Power spectrum of the $Y$ polarization and (b) polarization-resolved time series (blue: $X$; pink: $Y$ ) for $\Delta \nu=$ $-0.2 \mathrm{GHz}$ and $P_{\mathrm{inj}}=19.5 \mu \mathrm{W}$. The receiver is in the modal PS regime. The time series of $X$ polarization is shifted downward for visual convenience.

[22-25]. Type 2 instability only occurs when the parameters are close to the PS boundary, as shown in Fig. 7. Also the RF spectrum of Fig. 9(b) shows an enhancement of low-frequency component similar to that observed in VCSELs showing polarization mode hopping [24]. It also occurs as the signal from the transmitter is injected on the $\mathrm{TEM}_{10}$ mode. The third type of instability includes a low-frequency shoulder $(<1 \mathrm{GHz})$ and a sharp peak at $\sim 9 \mathrm{GHz}$ [Fig. 9(c)]. The low-frequency shoulder represents polarization competition between $X$ and $Y$ polarizations and is wider than that in type 2 instability. The magnitude of the $9 \mathrm{GHz}$ peak is affected by injection power. When the injection power is decreased but not too weak, the magnitude of the peak increases, as shown in Fig. 9 (d). Since the low-frequency shoulder is wide and there is a $\sim 9 \mathrm{GHz}$ peak in the power spectrum, it may indicate a transition to chaos via undamped relaxation oscillation. However, we did not obtain such a frequency of relaxation oscillation experimentally. Therefore the origin of this instability needs further investigation. Our theoretical modeling and numerical simulation are under way.

We also investigated whether there is any bistability for the observed PS since detuning-induced PS is typically accompanied by hysteresis regions in VCSELs [26]. When the frequency detuning is decreased, we observed a very narrow region of hysteresis. However, since the width of the region is similar to the level of reading uncertainty, we do not think that this is solid evidence of bistability. This may be related to the low power of the injected signal.

\section{DISCUSSION AND CONCLUSION}

It was reported that, when the VCSEL operates with two transverse modes-a fundamental mode and a first-order 

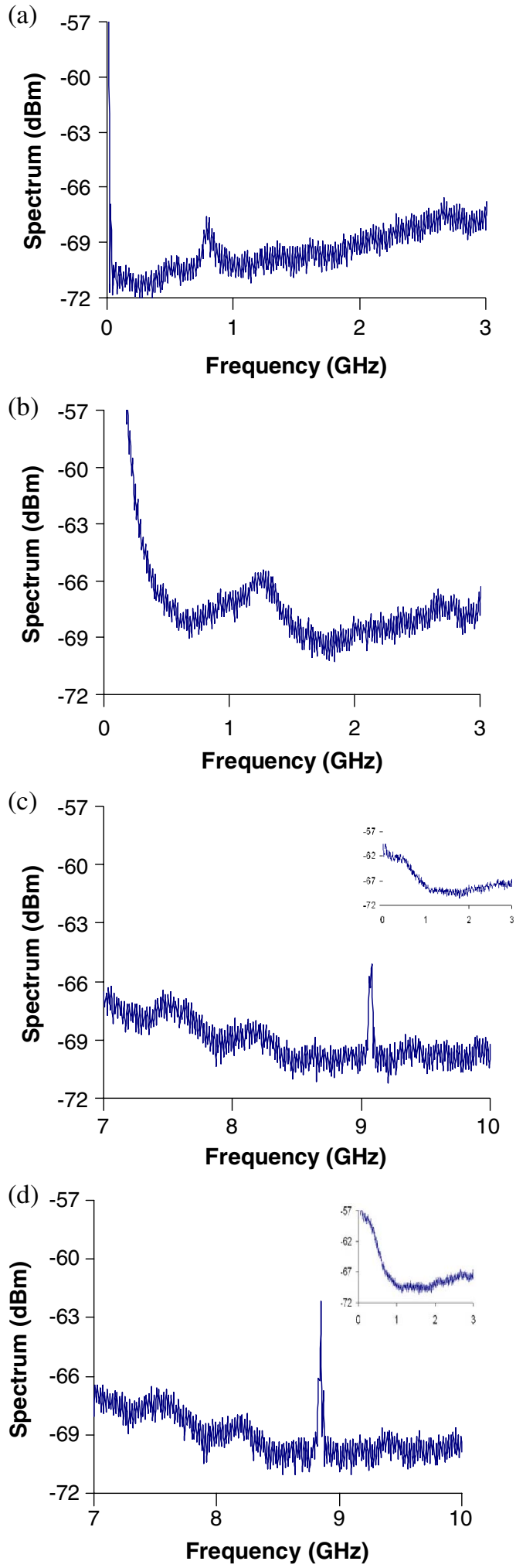

Fig. 9. (Color online) Power spectrum of the $Y$ polarization of $V_{1}$. (a) Type 1 instability for $\Delta \nu=-7.4 \mathrm{GHz}$, (b) type 2 instability for $\Delta \nu=-2 \mathrm{GHz}$, (c) type 3 instability for $\Delta \nu=0.4 \mathrm{GHz}$. The injection power is $19.5 \mu \mathrm{W}$. The inset shows the low-frequency shoulder in the power spectrum. (d) Type 3 instability for $P_{\text {inj }}=8.6 \mu \mathrm{W}$.

mode-with parallel polarization, the behaviors of the two modes are independent for both parallel and orthogonal optical injection. That is, optical injection only affects one mode while the other mode is unperturbed [5]. In our study, this modal independence was observed in Fig. 6 when the external signal is injected to the fundamental mode and the $\mathrm{TEM}_{10}$ mode, respectively. However, a strong modal correlation evidenced by PS in three modes was demonstrated when the $\mathrm{TEM}_{01}$ mode received the injection. The fourth mode, orthogonally polarized to the first three, is quite independent of the PS that occurs in the other modes. This implies that both spatial profile overlapping and modal strength play roles in the observed PS. When the external beam is injected into the $\mathrm{TEM}_{01}$ mode, the bias current of $V_{1}$ is more than two times the threshold. For this relatively high current, all the $X$-polarized transverse modes are strong and the $\mathrm{TEM}_{01}$ mode is the strongest one among them, as shown in Fig. 5 . Therefore, it is possible that the spatial coupling between the $\mathrm{TEM}_{01}$ mode and the other two modes (the $\mathrm{TEM}_{00}$ and $\mathrm{TEM}_{10}$ modes) are significantly stronger than their couplings for lower bias currents (Fig. 6) and this stronger coupling leads to the PS in all the three modes. The fourth transverse mode is much weaker than the first three modes, so its behavior is more independent. Our results also show that PS can be achieved with a very low injection power (a few microwatts), which is lower than most injected power used in previous studies [5, 11, 17]. This indicates a possible way to control the polarization of a multimode VCSEL via a weak signal.

The dynamics underlying the transition to polarization switching has been discussed for single $[\underline{12}, \underline{13}]$ and multimode $[10,11]$ VCSELs subject to orthogonal optical injection. Physics and bifurcations have been studied by doing a detailed analysis of the changes in optical spectra observed when changing the injected power for a fixed value of the frequency detuning. A better insight into the dynamics obtained in our experiment can be gained by comparing with results for multimode VCSELs $[10,11]$. Switching from the $X$ to the $Y$-polarized fundamental transverse mode is also obtained in [11] for low values of the injected power when the frequency of the optical injection is near to that of the high-order transverse mode. The transition to locked high-order transverse mode observed in [11] is not obtained in our experiment, possibly due to the limited injected power in our setup. However, a direct comparison with $[10,11]$ has its limitations because in our freerunning VCSEL three different modes have appreciable power whereas in $[10,11]$ only the fundamental mode of the solitary VCSEL is lasing. This difference suggests that an analysis of the dynamics, similar to that performed in [10-13], would be desirable in our system for a better understanding of the physics and bifurcations. But this analysis is beyond the scope of this paper.

In conclusion, we have studied experimentally the effect of a single-frequency orthogonal optical injection on a VCSEL operating with four transverse modes. Our results reveal that when the strongest mode, the $\mathrm{TEM}_{01}$ mode, is frequencylocked to the injection, polarization switching is achieved in the $\mathrm{TEM}_{01}$ mode as well as the other strong modes. This leads to polarization switching in the total output power of the receiver. The results imply that spatial overlapping and modal intensities play roles in the modal polarization switching in the fundamental mode and the $\mathrm{TEM}_{10}$ mode. We have identified three types of instabilities outside the modal polarization switching regime and proposed physical mechanisms for some of them. 


\section{ACKNOWLEDGMENTS}

This work is supported by the National Science Foundation under Grant No. PHY-1068789. A. Quirce and A. Valle thank support from the Spanish Research Council [Consejo Superior de Investigaciones Cientificas (CSIC)] and from Ministerio de Ciencia e Innovación, Spain, under project TEC2009-14581C02-02.

\section{REFERENCES}

1. D. Boggavarapu, R. Jin, J. Grantham, Y. Z. Hu, B. Colstoun, C. W. Lowry, G. Khitrova, S. W. Koch, M. Sargent III, H. M. Gibbs, and W. Chow, "Instabilities of a microcavity laser with a weak injected signal," Opt. Lett. 18, 1846-1848 (1993).

2. Z. G. Pan, S. Jiang, M. Dagenais, R. A. Morgan, K. Kojima, M. T. Asom, and R. E. Leibenguth, "Optical injection induced polarization bistability in vertical-cavity surface-emitting lasers," Appl. Phys. Lett. 63, 2999-3001 (1993).

3. H. Li, T. L. Lucas, J. G. McInerney, M. W. Wright, and R. A. Morgan, "Injection locking dynamics of vertical cavity semiconductor lasers under conventional and phase conjugate injection," IEEE J. Quantum Electron. 32, 227-235 (1996).

4. J. Y. Law, G. H. M. van Tartwijk, and G. P. Agrawal, "Effect of transverse-mode competition on the injection dynamics of vertical-cavity surface-emitting lasers," Quantum Semiclass. Opt. 9, 737-747 (1997).

5. Y. Hong, P. S. Spencer, P. Rees, and K. A. Shore, "Optical injection dynamics of two-mode vertical cavity surface-emitting semiconductor lasers," IEEE J. Quantum Electron. 38, 274-278 (2002).

6. Y. Hong, P. S. Spencer, S. Bandyoadhyay, P. Rees, and K. A. Shore, "Polarisation-resolved chaos and instabilities in a vertical-cavity surface-emitting laser subject to optical injection," Opt. Commun. 216, 185-189 (2003).

7. C.-H. Chang, L. Chrostowski, and C. J. Chang-Hasnain, "Injection locking of VCSELs," IEEE J. Sel. Top. Quantum Electron. 9, 1386-1393 (2003).

8. Y. Onishi, N. Nishiyama, C. Caneau, F. Koyama, and C. Zah, "Dynamic behavior of an all-optical inverter using transversemode switching in $1.55 \mathrm{~mm}$ vertical-cavity surface-emitting lasers," IEEE Photon. Technol. Lett. 16, 1236-1238 (2004).

9. X. Li, W. Pan, B. Luo, and D. Ma, "Multi-transverse-mode dynamics of vertical-cavity surface-emitting lasers with external optical injection,” J. Opt. Soc. Am. B 23, 1292-1301 (2006).

10. J. B. Altes, I. Gatare, K. Panajotov, H. Thienpont, and M. Sciamanna, "Mapping of the dynamics induced by orthogonal injection in vertical-cavity surface-emitting lasers," IEEE J. Quantum Electron. 42, 198-207 (2006).

11. A. Valle, I. Gatare, K. Panajotov, and M. Sciamanna, "Transverse mode switching and locking in vertical-cavity surface-emitting lasers subject to orthogonal optical injection," IEEE J. Quantum Electron. 43, 322-333 (2007).
12. M. Sciamanna and K. Panajotov, "Route to polarization switching induced by optical injection in vertical-cavity surfaceemitting lasers," Phys. Rev. A 73, 023811 (2006).

13. I. Gatare, M. Sciamanna, M. Nizette, and K. Panatojov, "Bifurcation to polarization switching and locking in vertical-cavity surface-emitting lasers with optical injection," Phys. Rev. A 76, 031803(R) (2007).

14. A. Hayat, A. Bacou, A. Rissons, J.-C. Mollier, V. Iakovlev, A. Sirbu, and E. Kapon, "Long wavelength VCSEL-by-VCSEL optical injection locking," IEEE Trans. Microwave Theor. Tech. 57, 1850-1858 (2009).

15. M. S. Torre, A. Valle, and L. Pesquera, "Transverse mode selection in vertical-cavity surface-emitting lasers with optical injection signal," IEEE J. Quantum Electron. 46, 105-111 (2010)

16. A. Hurtado, A. Quirce, A. Valle, L. Pesquera, and M. J. Adams, "Nonlinear dynamics induced by parallel and orthogonal injection in $1550 \mathrm{~nm}$ vertical-cavity surface-emitting lasers (VCSELs)," Opt. Express 18, 9423-9428 (2010).

17. P. Perez, A. Quirce, L. Pesquera, and A. Valle, "Polarizationresolved nonlinear dynamics induced by orthogonal optical injection in long-wavelength VCSELs," IEEE J. Sel. Top. Quantum Electron. 17, 1228-1235 (2011).

18. A. Quirce, J. R. Cuesta, A. Valle, A. Hurtado, L. Pesquera, and M. J. Adams, "Polarization bistability induced by orthogonal optical injection in $1550 \mathrm{~nm}$ multimode VCSELs," IEEE J. Sel. Top. Quantum Electron. 18, 772-778 (2012).

19. H. Lin, D. W. Pierce, A. Basnet, A. Quirce, Y. Zhang, and A. Valle, "Two-frequency injection on a multimode vertical-cavity surface-emitting laser," Opt. Express 19, 22437-22442 (2011).

20. M. Sondermann, M. Weinkath, T. Ackemann, J. Mulet, and S. Balle, "Two frequency emission and polarization dynamics at lasing threshold in vertical-cavity surface-emitting lasers," Phys. Rev. A 68, 033822 (2003)

21. J. Martin-Regalado, S. Balle, and M. San Miguel, "Polarization and transverse-mode dynamics of gain-guided vertical-cavity surface-emitting lasers," Opt. Lett. 22, 460-462 (1997).

22. M. B. Willemsen, M. U. F. Khalid, M.P. van Exter, and J. P. Woerdman, "Polarization switching of a vertical-cavity semiconductor laser as a Kramers hopping problem," Phys. Rev. Lett. 82, 4815-4818 (1999).

23. B. Nagler, M. Peeters, J. Albert, G. Verschaffelt, K. Panajotov, H. Thienpont, I. Veretennicoff, J. Danckaert, S. Barbay, G. Giacomelli, and F. Marin, "Polarization-mode hopping in single-mode vertical-cavity surface-emitting lasers: Theory and experiment," Phys. Rev. A 68, 013813 (2003).

24. G. Giacomelli and F. Marin, "Statistics of polarization competition in VCSELs," Quantum Semiclass. Opt. 10, 469-476 (1998).

25. J. Kaiser, C. Degen, and W. Elsässer, "Polarization switching influence on the intensity noise of vertical-cavity surface-emitting lasers," J. Opt. Soc. Am. B 19, 672-677 (2002).

26. I. Gatare, K. Panajotov, and M. Sciamanna, "Frequency-induced polarization bistability in vertical-cavity surface-emitting lasers with orthogonal optical injection," Phys. Rev. A 75, 023804 (2007). 